\title{
Why can states and measurement outcomes be represented as vectors?
}

\author{
Piero G. L. Mana* \\ Institutionen för Mikroelektronik och Informationsteknik (IMIT), \\ Royal Institute of Technology (KTH), \\ Electrum 229, SE-16440 Kista, Sweden
}

(Dated: 20 May 2003)

\begin{abstract}
It is shown how, given a "probability data table" for a quantum or classical system, the representation of states and measurement outcomes as vectors in a real vector space follows in a natural way. Some properties of the resulting sets of these vectors are discussed, as well as some connexions with the quantum-mechanical formalism.
\end{abstract}

PACS numbers: 03.65.Ta, 03.67.-a

\section{INTRODUCTION}

It has long been known that quantum mechanics is expressible through formalisms which differ, more or less, from the classical complex-Hilbert-space-based one; examples where given, among others, by Wigner [1], Stapp [2], Wootters [3, 4], Weigert [5], Hardy [6], Havel [7]. Some of these formalisms may be more useful than others in practical applications, but certainly all are very useful to understand better the physics "behind" quantum mechanics. This is particularly true when their mathematics is simple or, for example, geometrically appealing.

This is certainly the case for Hardy's formulation [6] of quantum mechanics in the simple language of real vector spaces.

Hardy's main idea, which was already expressed by Peres [8], is that a system's state, or preparation, is characterised by a list of "all probabilities for all measurements that could possibly be performed" on the system. However, such a list of probabilities is likely to be (infinite and) over-complete, "since most physical theories have some structure which relates different measured quantities"; discarding the "redundant" probabilities from the list, all that is left is simply a vector of real numbers: this is the state. An analogous conclusion can be drawn for the representation of measurement outcomes.

Such a framework is very general, and not restricted to the description of states and outcomes of quantummechanical systems only. Hardy characterises the latter by means of some simple, "reasonable" axioms.

A similar idea is proposed in the present paper, but from a slightly different perspective. It is shown that, given an experimental 'data table', containing statistical data about a generic classical or quantum system, the representation of preparations and measurement outcomes as vectors in a real vector space follows naturally as an alternative way of organising, or storing, the table's data. Some properties of the sets of these vectors are studied. The framework is general, not restricted to

\footnotetext{
*Electronic address: mana@imit.kth.se
}

quantum mechanics; connexions with the latter are discussed in the end.

\section{DECOMPOSITION OF A DATA TABLE}

Consider a system, which can be classical, quantum, or of unknown nature. It is easy to imagine the following: A physicist can prepare this system in a given number $M$ of different preparations, or states $\left\{s_{1}, \ldots, s_{M}\right\}$, and perform on it a given number of measurements $\left\{m_{k}\right\}$, each with a different number of outcomes $\left\{r_{i}: i \in I_{m_{k}}\right\}$ (mutually exclusive and exhaustive ${ }^{1}$ ), where the sets of indices $I_{m_{k}}$ depends on the measurement in question. The total number of outcomes of all measurements is $L$. Through experiments (and, possibly but not necessarily, some theoretical reasoning), the physicist can write down a table $\boldsymbol{p}$ with the probabilities for every outcome, for every measurement and state prepared; it may look like the following:

\begin{tabular}{cc|cccccc} 
& & $s_{1}$ & $s_{2}$ & $s_{3}$ & $s_{4}$ & $\ldots$ & $s_{M}$ \\
\hline$m_{1}$ & $r_{1}$ & $p_{11}$ & $p_{12}$ & $p_{13}$ & $p_{14}$ & $\ldots$ & $p_{1 M}$ \\
& $r_{2}$ & $p_{21}$ & $p_{22}$ & $p_{23}$ & $p_{24}$ & $\ldots$ & $p_{2 M}$ \\
\cline { 1 - 1 }$m_{2}$ & $r_{3}$ & $p_{31}$ & $p_{32}$ & $p_{33}$ & $p_{34}$ & $\ldots$ & $p_{3 M}$ \\
& $r_{4}$ & $p_{41}$ & $p_{42}$ & $p_{43}$ & $p_{44}$ & $\ldots$ & $p_{4 M}$ \\
& $r_{5}$ & $p_{51}$ & $p_{52}$ & $p_{53}$ & $p_{54}$ & $\ldots$ & $p_{5 M}$ \\
\cline { 1 - 1 }$m_{3}$ & $r_{6}$ & $p_{61}$ & $p_{62}$ & $p_{63}$ & $p_{64}$ & $\ldots$ & $p_{6 M}$ \\
& $\ldots$ & & & $\ldots$ & & \\
& $r_{L}$ & $p_{L 1}$ & $p_{L 2}$ & $p_{L 3}$ & $p_{L 4}$ & $\ldots$ & $p_{L M}$
\end{tabular}

The table, which can be called a '(experimental) probability data table' or 'data table' for short, has a column for every state and a row for every outcome; the table entry $(i, j)(e . g .,(4,2))$ is the probability $p_{i j}\left(p_{42}\right)$ of obtaining the outcome $r_{i}\left(r_{4}\right.$, among the possible outcomes $\left\{r_{3}, r_{4}, r_{5}\right\}$ of the measurement $\left.m_{2}\right)$ when the system is

\footnotetext{
1 This can always be achieved by grouping in suitable ways the outputs of the measurement, and adding if necessary the outcome "other".
} 
prepared in the state $s_{j}\left(s_{2}\right)$. States and outcomes can be listed and rearranged in any desired way in the table. Such a table would very likely have a large number of rows and columns, i.e., the numbers $L$ and $M$ are likely to be very large. ${ }^{2}$

Suppose that the physicist wants to find a more compact, or simply different, way to write down and store the data in $\boldsymbol{p}$. The table $\boldsymbol{p}$ is really just an $L \times M$ rectangular matrix, and as such it has a rank $K$, viz., the minimum number of linearly independent rows or columns:

$$
K \stackrel{\text { def }}{=} \operatorname{rank} \boldsymbol{p} \leqslant \min \{L, M\}
$$

It follows from linear algebra that $\boldsymbol{p}$ can be written as the product of an $L \times K$ matrix $\boldsymbol{t}$ and a $K \times M$ matrix $\boldsymbol{u}^{3}$ :

$$
p=t u
$$

or

$$
\begin{aligned}
&\left(\begin{array}{ccccc}
p_{11} & \ldots & p_{1 j} & \ldots & p_{1 M} \\
\ldots & \ldots & \ldots & \ldots & \ldots \\
p_{i 1} & \ldots & p_{i j} & \ldots & p_{i M} \\
\ldots & \ldots & \ldots & \ldots & \ldots \\
p_{L 1} & \ldots & p_{L j} & \ldots & p_{L M}
\end{array}\right)= \\
&=\left(\begin{array}{ccc}
t_{11} & \ldots & t_{1 K} \\
\ldots & \ldots & \ldots \\
\ldots & \ldots & t_{i K} \\
\ldots & \ldots & \ldots \\
t_{L 1} & \ldots & t_{L K}
\end{array}\right)\left(\begin{array}{ccccc}
u_{11} & \ldots & u_{1 j} & \ldots & u_{1 M} \\
\ldots & \ldots & \ldots & \ldots & \ldots \\
u_{K 1} & \ldots & u_{K j} & \ldots & u_{K M}
\end{array}\right) \\
&=\left(\begin{array}{c}
\boldsymbol{r}_{1}^{\top} \\
\ldots \\
\boldsymbol{r}_{i}^{\top} \\
\ldots \\
\boldsymbol{r}_{L}^{\top}
\end{array}\right)\left(\begin{array}{lllll}
\boldsymbol{s}_{1} & \ldots & \boldsymbol{s}_{j} & \ldots & \boldsymbol{s}_{M}
\end{array}\right)
\end{aligned}
$$

In the last equation, the matrix $\boldsymbol{t}$ has been written as a block of row vectors $\boldsymbol{r}_{i}^{\top}$, and the matrix $\boldsymbol{u}$ as a block of column vectors $\boldsymbol{s}_{i}$. In this decomposition, the element $p_{i j}$ of $\boldsymbol{p}$ is then given by the matrix product of the row vector $\boldsymbol{r}_{i}^{\top}$ with the column vector $\boldsymbol{s}_{j}$ :

$$
p_{i j}=\boldsymbol{r}_{i}^{\top} \boldsymbol{s}_{j}=\boldsymbol{r}_{i} \cdot \boldsymbol{s}_{j}, \quad \boldsymbol{r}_{i}, \boldsymbol{s}_{j} \in \mathbb{R}^{K},
$$

where, in the last expression, the row $\boldsymbol{r}_{i}$ and $\boldsymbol{s}_{j}$ are considered as vectors in $\mathbb{R}^{K}$, so that the matrix product is equivalent to the scalar product.

\footnotetext{
2 Of course, one may wonder how often a physicist has to concretely deal with similar tables, or whether a similar table has ever been written down actually; yet, it cannot be denied that this imaginary table conveys an idea of a part of that complex activity called "doing physics". Its main purpose here is to give a completely operational background to the concepts presented. It must also be remarked that it is not strictly necessary to speak about systems, states or preparations, and measurement outcomes: a similar table could be compiled by considering how different aspects of a given phenomenon are (cor)related to each other in their various manifestations. The terms 'system', 'state', 'measurement', and 'outcome' will nevertheless be used here for definiteness.

3 This is equivalent to the fact that a linear map $p: \mathbb{R}^{M} \rightarrow \mathbb{R}^{L}$ of rank $K \stackrel{\text { def }}{=} \operatorname{dim} p\left(\mathbb{R}^{M}\right)$ can be obtained as the composition $p=t \circ u$ of a surjective map $u: \mathbb{R}^{M} \rightarrow p\left(\mathbb{R}^{M}\right)$ and an injective $\operatorname{map} t: p\left(\mathbb{R}^{M}\right) \rightarrow \mathbb{R}^{L}$.
}

The result is that, given a probability table for a system, the states and the measurement outcomes for that system can be represented as vectors, $\left\{\boldsymbol{s}_{j}\right\}$ and $\left\{\boldsymbol{r}_{i}\right\}$, in $\mathbb{R}^{K}$, for some $K$, and the relative probabilities are given by their scalar product. These vectors can be called state vectors and outcome vectors. As already said, this kind of representation has already been presented by Hardy [6] through a different line of reasoning. In particular, Hardy supposes that it is possible to represent a state with a $K$ dimensional vector ${ }^{4}$, with $K \leqslant L$, because "most physical theories have some structure which relates different measured quantities"; but the reasoning above shows that this possibility exists even before building up some theory to describe the data.

The matrices $\boldsymbol{t}$ and $\boldsymbol{u}$ are not uniquely determined from the decomposition (2), so that there is some freedom in choosing their form. The fact that $\operatorname{rank} \boldsymbol{p}=K$, implies that there exists a square $K \times K$ submatrix $\boldsymbol{a}$, obtained from $\boldsymbol{p}$ by suppressing $(L-K)$ rows and $(M-K)$ columns, such that $\operatorname{det} \boldsymbol{a} \neq 0$. It is always possible to rearrange the rows and the columns of the table $\boldsymbol{p}$ so that such submatrix is the one formed by the first $K$ rows and $K$ columns. After this rearrangement (which, of course, does not imply any physical operation on the system), $\boldsymbol{p}$ can be written in the following block form:

$$
\boldsymbol{p}=\left(\begin{array}{ll}
\boldsymbol{a} & \boldsymbol{b} \\
\boldsymbol{c} & \boldsymbol{d}
\end{array}\right) \quad \text { with } \operatorname{det} \boldsymbol{a} \neq 0,
$$

where $\boldsymbol{b}, \boldsymbol{c}$, and $\boldsymbol{d}$ are of order $K \times(M-K),(L-K) \times K$, and $(L-K) \times(M-K)$ respectively.

By writing also the matrices $\boldsymbol{t}$ and $\boldsymbol{u}$ in block form

$$
\boldsymbol{t}=\left(\begin{array}{c}
\boldsymbol{v} \\
\boldsymbol{w}
\end{array}\right), \quad \boldsymbol{u}=\left(\begin{array}{ll}
\boldsymbol{x} & \boldsymbol{y}
\end{array}\right),
$$

where the orders of $\boldsymbol{v}, \boldsymbol{w}, \boldsymbol{x}$, and $\boldsymbol{y}$ are $K \times K,(L-K) \times K$, $K \times K$, and $K \times(M-K)$ respectively, we can rewrite Eq. (2) as

$$
\left(\begin{array}{ll}
a & b \\
c & d
\end{array}\right)=\left(\begin{array}{c}
v \\
w
\end{array}\right)\left(\begin{array}{ll}
x & y
\end{array}\right), \quad \text { or } \quad\left\{\begin{array}{l}
a=v x \\
b=v y \\
c=w x \\
d=w y
\end{array}\right.
$$

with the solution ${ }^{5}$

$$
\left\{\begin{array} { r l } 
{ \boldsymbol { v } } & { = \boldsymbol { a } \boldsymbol { x } ^ { - 1 } } \\
{ \boldsymbol { w } } & { = \boldsymbol { c } \boldsymbol { x } ^ { - 1 } } \\
{ \boldsymbol { y } } & { = \boldsymbol { x } \boldsymbol { a } ^ { - 1 } \boldsymbol { b } } \\
{ \boldsymbol { d } } & { = \boldsymbol { c } \boldsymbol { a } ^ { - 1 } \boldsymbol { b } }
\end{array} \text { or } \quad \left\{\begin{array}{rl}
\boldsymbol{t} & =\left(\begin{array}{l}
\boldsymbol{a} \boldsymbol{x}^{-1} \\
\boldsymbol{c} \boldsymbol{x}^{-1}
\end{array}\right) \\
\boldsymbol{u} & =\left(\begin{array}{ll}
\boldsymbol{x} & \boldsymbol{x} \boldsymbol{a}^{-1} \boldsymbol{b}
\end{array}\right)
\end{array} \quad \operatorname{det} \boldsymbol{x} \neq 0,\right.\right.
$$

\footnotetext{
${ }^{4}$ Hardy calls $K$ the number of degrees of freedom of the system.

5 The submatrix $\boldsymbol{d}$ of $\boldsymbol{p}$ is completely determined by the other submatrices $\boldsymbol{a}, \boldsymbol{b}$, and $\boldsymbol{c}$ because rank $\boldsymbol{p}=K$.
} 
where the square matrix

$$
\boldsymbol{x}=\left(\begin{array}{lll}
\boldsymbol{s}_{1} & \ldots & \boldsymbol{s}_{K}
\end{array}\right)
$$

is undetermined except for the condition of being nonsingular; this corresponds to the freedom of choosing $K$ basis vectors in $\mathbb{R}^{K}$ as the representatives of the first $K$ states $^{6}\left\{\boldsymbol{s}_{1}, \ldots, \boldsymbol{s}_{K}\right\}$, which can then be called basis states.

\section{THE SETS OF STATES AND OUTCOMES}

The vectors by which states and outcomes are represented belong to two subsets, $S \stackrel{\text { def }}{=}\left\{s_{1}, \ldots, s_{M}\right\}$ and $R \stackrel{\text { def }}{=}\left\{\boldsymbol{r}_{1}, \ldots, \boldsymbol{r}_{L}\right\}$ respectively, of $\mathbb{R}^{K}$. It is interesting to study some properties of these sets. Some of the following results have been obtained by Peres and Terno [9] in the framework of quantum mechanics. The convex-related properties of the sets are also well known $[10,11,12$, and references therein], but are often derived and expressed through more elegant, and abstract, mathematics.

In the following, the terms 'vector' and 'point' are used interchangeably.

\section{A. "Completion" of the sets}

One can consider the convex hull of the set of states $S$ :

$$
S_{\mathrm{c}} \stackrel{\text { def }}{=}\left\{\sum_{i=1}^{M} \lambda_{i} \boldsymbol{s}_{i} \mid \boldsymbol{s}_{i} \in S, \lambda_{i} \geqslant 0, \sum_{i=1}^{M} \lambda_{i}=1\right\} .
$$

An element of $S_{\mathrm{c}}$ like, e.g., $\lambda \boldsymbol{s}_{j^{\prime}}+(1-\lambda) \boldsymbol{s}_{j^{\prime \prime}}$, with $\boldsymbol{s}_{j^{\prime}}, \boldsymbol{s}_{j^{\prime \prime}} \in S$ (and $0 \leqslant \lambda \leqslant 1$ ), can be considered as a possible state, corresponding to a preparation in which $\boldsymbol{s}_{j^{\prime}}$ or $\boldsymbol{s}_{j^{\prime \prime}}$ are chosen with probabilities $\lambda$ or $(1-\lambda)$ respectively. This state should then be added to the table $\boldsymbol{p}$, with a respective column of probabilities. However, this column would be, for obvious reasons, just a linear combination of the columns under $\boldsymbol{s}_{j^{\prime}}$ and $\boldsymbol{s}_{j^{\prime \prime}}$, with coefficients $\lambda$ and $(1-\lambda)$; as a consequence, the rank of the table would be still $K$. For this reason, the set $S$ and its convex hull $S_{\mathrm{c}}$ can be used interchangeably in the considerations to follow ${ }^{7}$. Reasoning in terms of convexity, one sees that $S_{\mathrm{c}}$ is just a convex (not necessarily regular) polytope in a $(K-1)$-dimensional (Euclidean) space (for example, a triangle, rhomboid, or general polygon in

\footnotetext{
6 There is the alternative option of choosing the representatives of the first $K$ outcomes; this corresponds to solving Eq. (7) in terms of $\boldsymbol{v}$.

7 This corresponds to "completing" the table $\boldsymbol{p}$ with this (infinite) number of "additional" states; so $M$ tends to infinity, but the rank $K$ remains constant.
}

two dimensions; or a tetrahedron, cube, prism, or general polyhedron in three, or a 600-cell in four, and so on [13]). Some of the states $\left\{\boldsymbol{s}_{1}, \ldots, \boldsymbol{s}_{M}\right\}$ are extreme points of this convex set (vertices of the polytope). Since every state vector can be written as a convex, hence linear, combination of these extreme states, they must be at least as numerous as the basis states. The number $Z$ of extreme states (in quantum mechanics, they are called pure states) must then satisfy

$$
Z \geqslant K
$$

For the set of outcomes, the situation is slightly different, and convex combination is not the only way in which outcomes can be combined. Consider, as a concrete example, the state $\boldsymbol{s}_{j}$ and the measurements $m^{\prime}$, $m^{\prime \prime}$, and $m^{\prime \prime \prime}$ with outcomes $\left\{\boldsymbol{r}_{1}, \boldsymbol{r}_{2}, \boldsymbol{r}_{3}\right\},\left\{\boldsymbol{r}_{4}, \boldsymbol{r}_{5}\right\}$, and $\left\{\boldsymbol{r}_{6}, \boldsymbol{r}_{7}\right\}$ respectively, and the corresponding probabilities; from them, the following additional measurements can be derived:

- The measurement which consists in performing $m^{\prime}$ but considering only the set of two results $\left\{\left(\boldsymbol{r}_{1}\right.\right.$ or $\left.\left.\boldsymbol{r}_{3}\right), \boldsymbol{r}_{2}\right\}$, with probabilities $\left\{\left(p_{1 j}+p_{3 j}\right), p_{2 j}\right\}$ (a sort of coarse-graining).

- The measurement which consistes in performing $m^{\prime}$ with probability $\lambda^{\prime}$, or $m^{\prime \prime}$ with probability $\lambda^{\prime \prime}$, or $m^{\prime \prime \prime}$ with probability $\lambda^{\prime \prime \prime}$ (obviously, $\left.\lambda^{\prime}+\lambda^{\prime \prime}+\lambda^{\prime \prime \prime}=1\right)$. The experimenter (who may not know which measurement will actually be performed) expects thus one of the outcomes $\left\{\boldsymbol{r}_{1}, \boldsymbol{r}_{2}, \boldsymbol{r}_{3}, \boldsymbol{r}_{4}, \boldsymbol{r}_{5}, \boldsymbol{r}_{6}, \boldsymbol{r}_{7}\right\}$ with probabilities $\left\{\lambda^{\prime} p_{1 j}, \lambda^{\prime} p_{2 j}, \lambda^{\prime} p_{3 j}, \lambda^{\prime \prime} p_{4 j}, \lambda^{\prime \prime} p_{5 j}, \lambda^{\prime \prime \prime} p_{6 j}, \lambda^{\prime \prime \prime} p_{7 j}\right\}$ (of course, obtaining, e.g., the result $\boldsymbol{r}_{2}$ would imply that $m^{\prime}$ was actually performed).

- Combinations of the two cases above.

From the examples just given, it is easy to see that, given two outcomes $\boldsymbol{r}_{i^{\prime}}$ and $\boldsymbol{r}_{i^{\prime \prime}}$, not necessarily of the same measurement, one can consider also the outcomes

$$
\lambda^{\prime} \boldsymbol{r}_{i^{\prime}}+\lambda^{\prime \prime} \boldsymbol{r}_{i^{\prime \prime}} \quad\left(0 \leqslant \lambda^{\prime}+\lambda^{\prime \prime} \leqslant 1 \text { and } \lambda^{\prime}, \lambda^{\prime \prime} \geqslant 0\right),
$$

and

$$
\boldsymbol{r}_{i^{\prime}}+\boldsymbol{r}_{i^{\prime \prime}} \quad\left(\text { only if } m^{\prime}=m^{\prime \prime}\right)
$$

Note that Eq. (12) is not (always) a convex combination. The null vector (origin) $\boldsymbol{o}$ belongs thus to the set $R_{\mathrm{c}}$.

These measurements and outcomes could be added to the table $\boldsymbol{p}$ as well, with their relative rows of probabilities; the rank of the table would nevertheless remain $K$ for the same reason given for the additional states. Thus, also the set of outcomes $R$ can be ideally extended to a set $R_{\mathrm{c}}$ by means of Eqs. (12) and (13). Again, $R$ and $R_{\mathrm{c}}$ will be referred to interchangeably in the following. 


\section{B. The set of states lies in a hyperplane}

Consider a state $\boldsymbol{s}_{j} \in S$ and all the outcomes $\left\{\boldsymbol{r}_{i^{\prime}}: i^{\prime} \in\right.$ $\left.I_{m^{\prime}}\right\}$ of a given measurement $m^{\prime}$, where the sets of indices $I_{m^{\prime}}$ depends on $m^{\prime}$. Since the outcomes are exhaustive and mutually exclusive, their probabilities must sum up to unity:

$$
\sum_{i^{\prime} \in I_{m^{\prime}}} p_{i^{\prime} j}=\sum_{i^{\prime} \in I_{m^{\prime}}}\left(\boldsymbol{r}_{i^{\prime}} \cdot \boldsymbol{s}_{j}\right)=\left(\sum_{i^{\prime} \in I_{m^{\prime}}} \boldsymbol{r}_{i^{\prime}}\right) \cdot \boldsymbol{s}_{j}=1 .
$$

The equation above, when considered for the first $K$ states $\left\{\boldsymbol{s}_{1}, \ldots, \boldsymbol{s}_{K}\right\}$, which form a basis for $\mathbb{R}^{K}$, uniquely determines the vector sum of the outcomes of $m^{\prime}$, denoted by $\boldsymbol{n}^{\prime} \stackrel{\text { def }}{=} \sum_{i^{\prime} \in I_{m^{\prime}}}, \boldsymbol{r}_{i^{\prime}}$, by its projections along the basis vectors. On the other hand, this happens for the outcomes of any measurement $m$. Hence, the sum of the outcome vectors $\left\{\boldsymbol{r}_{i}: i \in I_{m}\right\}$ of any measurement $m$ is a constant vector

$$
\boldsymbol{n} \equiv \sum_{i \in I_{m}} \boldsymbol{r}_{i}, \quad \text { for any } m,
$$

such that

$$
\boldsymbol{n}^{\top} \boldsymbol{s} \equiv \boldsymbol{n} \cdot \boldsymbol{s}=1 .
$$

The vector $\boldsymbol{n}$ (which is an extreme point of $R_{\mathrm{c}}$ ) may be called the trivial-measurement vector, since it also represents the outcome of the trivial measurement having only a single (and therefore certain) outcome for any state. The actual components of this vector are determined by the choice of the matrix $\boldsymbol{x}$. In fact, Eq. (16) holds, in particular, for every basis state $\left\{\boldsymbol{s}_{1}, \ldots, \boldsymbol{s}_{K}\right\}$, and the set of these $K$ equations can be written, with the help of formula (9), in the following matrix form:

$$
\boldsymbol{n}^{\top} \boldsymbol{x}=\boldsymbol{q}^{\top}, \quad \text { with } \boldsymbol{q}=(\overbrace{\left.\begin{array}{llll}
1 & 1 & \ldots & 1
\end{array}\right)}^{K \text { elements }} .
$$

Since $\boldsymbol{x}$ is non-singular, one finds

$$
\boldsymbol{n}^{\top}=\boldsymbol{q}^{\top} \boldsymbol{x}^{-1}
$$

so that $\boldsymbol{x}$ determines $\boldsymbol{n}$, as asserted.

Finally, the equation (16), where $\boldsymbol{n}$ is now considered constant, must be satisfied by every state $s$; this is the equation, in vector form, of a (affine) hyperplane normal to $\boldsymbol{n}$. Hence, the set of states $S$ lies in a $(K-1)$ dimensional (affine) hyperplane in $\mathbb{R}^{K}$.

\section{The set of states determines the maximal possible extension of the set of outcomes}

The sets $S$ and $R$ can thus be viewed as compact, convex regions in $\mathbb{R}^{K}$. Their boundaries are interrelated ${ }^{8}$.

\footnotetext{
8 They are dual [10].
}

In particular, it is interesting to study how the boundary of $S$ determines the region wherein $R$ is constrained to lie (though $R$ may be a proper subset of this region).

Since $0 \leqslant p \leqslant 1$ for every probability $p=\boldsymbol{r} \cdot \boldsymbol{s}$, one has

$$
0 \leqslant \boldsymbol{r} \cdot \boldsymbol{s} \leqslant 1
$$

The formula above, with $s$ considered constant and $\boldsymbol{r}$ variable, defines a region in $\mathbb{R}^{K}$ delimited by the parallel hyperplanes $\boldsymbol{r} \cdot \boldsymbol{s}=0$ and $\boldsymbol{r} \cdot \boldsymbol{s}=1$. These hyperplanes are both perpendicular to the vector $s$, and pass respectively through the origin $\boldsymbol{o}$ and through the point $\boldsymbol{n}$, since $\boldsymbol{n} \cdot \boldsymbol{s}=$ 1 by Eq. (16). There is one such region for every state $s \in S$. The outcome vectors must be confined to lie in the intersection of all these regions. However, if an outcome vector $\boldsymbol{r}$ lies inside the regions determined by two states $\boldsymbol{s}^{\prime}$ and $\boldsymbol{s}^{\prime \prime}$, it must also lie in that determined by any state $\lambda \boldsymbol{s}^{\prime}+(1-\lambda) \boldsymbol{s}^{\prime \prime}$ which is their convex combination; i.e., if $0 \leqslant \lambda \leqslant 1$, then

$$
\left.\begin{array}{l}
0 \leqslant \boldsymbol{r} \cdot \boldsymbol{s}^{\prime} \leqslant 1 \\
0 \leqslant \boldsymbol{r} \cdot \boldsymbol{s}^{\prime \prime} \leqslant 1
\end{array}\right\} \Rightarrow 0 \leqslant \boldsymbol{r} \cdot\left[\lambda \boldsymbol{s}^{\prime}+(1-\lambda) \boldsymbol{s}^{\prime \prime}\right] \leqslant 1 .
$$

This is easily proven observing that, since $\boldsymbol{r} \cdot \boldsymbol{s}^{\prime}, \boldsymbol{r} \cdot \boldsymbol{s}^{\prime \prime}, \lambda$, and $(1-\lambda)$ are non-negative, then $\lambda \boldsymbol{r} \cdot \boldsymbol{s}^{\prime}+(1-\lambda) \boldsymbol{r} \cdot \boldsymbol{s}^{\prime \prime} \geqslant 0$; and since $\boldsymbol{r} \cdot \boldsymbol{s}^{\prime}, \boldsymbol{r} \cdot \boldsymbol{s}^{\prime \prime} \leqslant 1$, then $\lambda \boldsymbol{r} \cdot \boldsymbol{s}^{\prime}+(1-\lambda) \boldsymbol{r} \cdot \boldsymbol{s}^{\prime \prime} \leqslant$ $\lambda+(1-\lambda)=1$.

Hence, one needs to consider only the regions determined by the $Z$ extreme states, which are the only ones not expressible as convex combinations of other states. Now let $\left\{\boldsymbol{s}_{i_{1}}, \ldots, \boldsymbol{s}_{i_{Z}}\right\}$ be the extreme states. Consider the $Z$ hyperplanes of equations $\boldsymbol{r} \cdot \boldsymbol{s}_{i_{j}}=0, j=1, \ldots, Z$ : they delimit a convex cone with vertex in the origin $\boldsymbol{o}$. Another convex cone, with vertex in $\boldsymbol{n}$, is delimited by the other $Z$ hyperplanes of equations $\boldsymbol{r} \cdot \boldsymbol{s}_{i_{j}}=1$, $j=1, \ldots, Z$. The intersection of these two cones ${ }^{9}$ finally determines the maximal, $K$-dimensional, convex region which can be occupied by the set of outcomes $R$.

\section{D. 'One-shot' distinguishability}

Suppose that there exists a measurement $m$ that allows one to tell with certainty which state, from a given set of $D$ states $\left\{\boldsymbol{s}_{j_{1}}, \ldots, \boldsymbol{s}_{j_{D}}\right\}$, is actually prepared. These states can then be called 'one-shot distinguishable'. This means that the table $\boldsymbol{p}$ must contain a subtable of the form (obtained, if necessary, by re-listing states and outcomes):

\footnotetext{
${ }^{9}$ It can be shown that the cones are symmetric with respect to the point (center of symmetry) $\boldsymbol{n} / 2$ : indeed, if $\boldsymbol{r} \cdot \boldsymbol{s}=0$ or 1 for some $\boldsymbol{r}$ and $\boldsymbol{s}$, then $[\boldsymbol{n} / 2+(\boldsymbol{n} / 2-\boldsymbol{r})] \cdot \boldsymbol{s} \equiv \boldsymbol{n} \cdot \boldsymbol{s}-\boldsymbol{r} \cdot \boldsymbol{s}=1-\boldsymbol{r} \cdot \boldsymbol{s}=1$ or 0 , where $\boldsymbol{n} / 2+(\boldsymbol{n} / 2-\boldsymbol{r})$ is the point symmetric to $\boldsymbol{r}$ with respect to $\boldsymbol{n} / 2$.
} 


\begin{tabular}{rr|cccc} 
& & $s_{i_{1}}$ & $s_{i_{2}}$ & $\ldots$ & $s_{i_{D}}$ \\
\hline$m r_{i_{1}}$ & 1 & 0 & $\ldots$ & 0 \\
$r_{i_{2}}$ & 0 & 1 & $\ldots$ & 0 \\
$\ldots$ & & $\ldots$ & \\
$r_{i_{D}}$ & 0 & 0 & $\ldots$ & 1
\end{tabular}

or, in other words, the matrix $\boldsymbol{p}$ has a square $D \times D$ submatrix equal to the $D \times D$ identity matrix $\boldsymbol{E}_{D}$. This implies that

$$
\operatorname{rank} \boldsymbol{p} \equiv K \geqslant D \text {. }
$$

It can be proven that, if some of the states $\left\{\boldsymbol{s}_{j_{1}}, \ldots, \boldsymbol{s}_{j_{D}}\right\}$ are not extreme, they can be replaced by extreme states having the same distinguishability property [6, Sec. 6.12]. Moreover, if the $\left\{\boldsymbol{s}_{j_{1}}, \ldots, \boldsymbol{s}_{j_{D}}\right\}$ are one-shot distinguishable extreme states, then the convex combination of any $(D-1)$ of them must lie on the boundary of $S$; a simple proof of this fact for the case of three one-shot distinguishable extreme states is given in Appendix A. This implies that, if $S$ contains $D$ oneshot distinguishable states, there must exist a $(D-1)$ dimensional hyperplane such that its intersection with $S$ is a $(D-1)$-dimensional simplex.

In particular, this is also true for the set of states in quantum mechanics. For example, the set of states of a three-level system, which has at most three distinguishable states, shows triangular two-dimensional sections [14, Fig. 2]; for a four-level system, a threedimensional section of the set of states yields a tetrahedron, and so some two-dimensional sections must have triangular as well as trapezoidal shapes [15, Fig. 1].

The maximum number of one-shot distinguishable states for a given system with table $\boldsymbol{p}$ will be denoted by $N ;{ }^{10}$ from Eqs. (11) and (21) it follows that

$$
Z \geqslant K \geqslant N
$$

Note that, if $K=N$, then $Z=K \equiv N$ as well. This is because the $N$ distinguishable states can be chosen to be extreme, and then they are the vertices of a $(N-1)$ dimensional simplex; but this must be all of $S$, since $S$ is $(N-1$ )-dimensional (for $K=N$ ) and convex. (In this case, $R$ is a $K$-dimensional hypercube.)

\section{MAPS BETWEEN SETS OF STATES}

There is, of course, more than just one system; the transformations and relations among systems (like the relation system-subsystem) are extremely important.

A transformation or relation between a system with table $\boldsymbol{p}^{\prime}$ and another with table $\boldsymbol{p}^{\prime \prime}$ can be in some cases expressed by means of a map $S^{\prime} \rightarrow S^{\prime \prime}$ between the set

\footnotetext{
${ }^{10}$ Hardy calls $N$ the dimension of the system.
}

of states $S^{\prime} \in \mathbb{R}^{K^{\prime}}$ of $\boldsymbol{p}^{\prime}$, and the set of states $S^{\prime \prime} \in \mathbb{R}^{K^{\prime \prime}}$ of $\boldsymbol{p}^{\prime \prime}$. The map should preserve convex combinations of state vectors ${ }^{11}$; this implies that its most general form is that of an affine map, which can be written as

$$
\boldsymbol{s}^{\prime} \mapsto \boldsymbol{s}^{\prime \prime}=\boldsymbol{F} \boldsymbol{s}^{\prime}+\boldsymbol{g},
$$

where $\boldsymbol{F}$ is a $K^{\prime \prime} \times K^{\prime}$ matrix and $\boldsymbol{g} \in \mathbb{R}^{K^{\prime \prime}}$ a column vector.

It turns out, however, that such a map is always expressible as a linear transformation between the state vectors in $S^{\prime}$ and $S^{\prime \prime}$. This is due to the fact that $S^{\prime}$ lies in an affine hyperplane of $\mathbb{R}^{K^{\prime}}$, and is simply proven by considering Eq. (16) for the set $S^{\prime}$ :

$$
n^{\prime \top} s^{\prime}=1, \quad s^{\prime} \in S^{\prime},
$$

where $\boldsymbol{n}^{\prime}$ is the trivial-measurement vector of $R^{\prime}$, and then defining

$$
\boldsymbol{C} \stackrel{\text { def }}{=} \boldsymbol{F}+\boldsymbol{g} \boldsymbol{n}^{\prime \mathrm{T}},
$$

which is a $K^{\prime \prime} \times K^{\prime}$ matrix representing a linear transformation. By Eq. (24) one finds

$$
\boldsymbol{C} \boldsymbol{s}^{\prime}=\boldsymbol{F} \boldsymbol{s}^{\prime}+\boldsymbol{g n ^ { \prime \top }} \boldsymbol{s}^{\prime}=\boldsymbol{F} \boldsymbol{s}^{\prime}+\boldsymbol{g}
$$

as asserted.

Every such a map $C$ between states induces a dual map from the set, $R^{\prime \prime} \in \mathbb{R}^{K^{\prime \prime}}$, of outcomes of $\boldsymbol{p}^{\prime \prime}$ to that, $R^{\prime} \in \mathbb{R}^{K^{\prime}}$, of $\boldsymbol{p}^{\prime}$ :

$$
\boldsymbol{r}^{\prime \prime \top} \mapsto \boldsymbol{r}^{\prime \top}=\boldsymbol{r}^{\prime \prime \top} C, \quad \text { or } \quad \boldsymbol{r}^{\prime \prime} \mapsto \boldsymbol{r}^{\prime}=\boldsymbol{C}^{\top} \boldsymbol{r}^{\prime \prime} .
$$

The dual map must, for obvious reasons, map the trivialmeasurement vector $\boldsymbol{n}^{\prime \prime}$ of $R^{\prime \prime}$ to the trivial-measurement vector $\boldsymbol{n}^{\prime}$ of $R^{\prime}$ :

$$
\boldsymbol{C}^{\top} \boldsymbol{n}^{\prime \prime}=\boldsymbol{n}^{\prime}
$$

Which represents a constraint on the possible form of $\boldsymbol{C}$.

An analysis of the characteristics of the set of possible maps between system states, analogous to that conducted on the sets $S$ and $R$, would be very interesting, but it will not be pursued here. Such analysis could offer new perspectives (complementary to the 'tensor-product based' one) to study the relation system-subsystem ${ }^{12}$ and the question of the complete positivity of superoperators $[16,17,18,19]$.

11 This map, however, could in principle be partial, i.e., not defined for all $\boldsymbol{s}^{\prime} \in S^{\prime}$; this is because there may be, e.g., a transformation device that cannot take just any state of $S^{\prime}$ as input.

12 The fact that $p^{\prime \prime}$ is a subsystem of $p^{\prime \prime}$ can be expressed by saying that there exists a surjective, non-injective map from $S^{\prime}$ to $S^{\prime \prime}$. Intuitively, this is because every preparation of a system is also a preparation for one of its subsystems, and a preparation of a subsystem may correspond to different preparations of the system it is part of. 


\section{HARDY'S AXIOMATICS AND QUANTUM MECHANICS}

The formalism just presented is quite general, containing the quantum mechanical one as a particular case (the relation with the trace rule is quickly shown in Appendix B), and is essentially the same as Hardy's, prior to the introduction of his axioms. The effect of the latter [6] is to characterise classical and quantum systems by means of constraints on the set of states and that of outcomes.

A classical system is characterised, in Hardy's axiomatics, by the fact that the number of one-shot distinguishable states is maximal, i.e., $N=K$; as a consequence, the number of extreme states is $Z=K=N$ as well, as shown in Sec. III D.

A quantum mechanical system, instead, is characterised by the supposed existence of continuous reversible transformations between extreme states ${ }^{13}$. This implies that the extreme vectors of a quantum mechanical set of states $S_{\mathrm{QM}}$ form a continuum $(Z=\operatorname{card} \mathbb{R})$; they satisfy also a quadratic equation which determines the "shape" (modulo isomorphisms, in the convex sense) of $S_{\mathrm{QM}}$. Moreover, the maximal number of one-shot distinguishable states is characterised by $K=N^{2}$. Some remarks can be made on these features.

A continuum of extreme states can never be observed in practice [22, Chap. 9], but is a useful approximation or inductive generalisation which makes the powerful tools of analysis available for doing physics. The same approximative or inductive step is indeed taken also in classical physics (think of classical phase space): in this case the distinguishable states, and hence the extreme states as well, are supposed to form a continuum $(N=Z=\operatorname{card} \mathbb{R})$. From this point of view the continuum assumption of quantum mechanics is more economical than the one of classical physics.

On the other hand, it is to be noted that most (if not all) typical features of quantum mechanics arise not from the continuity of the extreme states, but from the fact that there are more extreme states than distinguishable ones, i.e., from $Z>N$ (which implies $K>N$ ). This can be seen from the examples given by Kirkpatrick [23, 24, 25] (see also Ref. 26). Note, however, that in those examples one finds that $K \neq N^{2}$, and $Z$, $K, N$ are finite. ${ }^{14}$

It is reasonable to ask, from this point of view, to what extent the above-mentioned quantum mechanical constraints on the sets of states and outcomes are actually observed in practice. There are cases in which

13 Hardy adopts the old saying "natura in operationibus suis non facit saltum" as expressed by Tissot [20] (see also von Linné [21]).

14 It would be interesting to develop, and study the properties of, a general mathematical formalism having no constraints on the sets $S$ and $R$ or on the numbers $Z, K, N$, and thus containing the classical and the quantum-mechanical as special cases. they are not; for example, in the presence of superselection rules, where, roughly speaking, some "portions" of the quantum mechanical set of states $S_{\mathrm{QM}}$ are actually missing, i.e., are not observed (the same happens for the quantum mechanical set of outcomes $R_{\mathrm{QM}}$ ).

A related question is whether, given a generic probability data table $\boldsymbol{p}$, the set of states $S$ and the set of outcomes $R$ derived from it can be "embedded" in some larger sets $S_{\mathrm{QM}}$ and $R_{\mathrm{QM}}$ satisfying the quantum mechanical constraints. If this were always possible, then quantum mechanics would be "always right", just because every every experimental data table could then always be described by quantum mechanical means.

\section{DISCUSSION}

Using the conceptual tool of an imaginary 'probability data table' associated to a system, it has been shown that the states and measurement outcomes of the system can be represented as vectors in a real vector space. This representation simply follows from the decomposition of the table, and in this context the rank of the latter, $K$, has a peculiar rôle. This framework is very similar in spirit to Hardy's framework before the introduction of his axioms, and may thus elucidate some features of the latter.

Some properties of the sets of states and outcomes, for a generic classical or quantum mechanical system, have then been analysed in simple geometrical terms.

Finally, some points have been discussed concerning the characteristics of the sets of states of a quantum mechanical system as formalised by Hardy. In particular, it has been argued that the origin of many typical quantum mechanical features lies not specifically in the continuity of the extreme states, or in the relation $K=N^{2}$ between $K$ and the number of 'one-shot distinguishable' states $N$, but simply in the fact that the number of extreme states is greater than the number of 'one-shot distinguishable' states.

\section{Acknowledgments}

The author would like to thank Professor Gunnar Björk for advice and useful discussions, and Åsa Ericsson for a useful discussion.

\section{Appendix A}

In Sec. IIID it was stated that, if $\left\{\boldsymbol{s}_{j_{1}}, \ldots, \boldsymbol{s}_{j_{D}}\right\}$ are one-shot distinguishable extreme states, then the convex combination of any $(D-1)$ of them must lie on the boundary of $S$. A simple proof for three one-shot distinguishable extreme states $\left\{\boldsymbol{s}_{j_{1}}, \boldsymbol{s}_{j_{2}}, \boldsymbol{s}_{j_{3}}\right\}$ is the following.

First, the condition for a point $s \in S$ to belong to the boundary is that there exists at least one point $\boldsymbol{s}_{*} \in S$, 
$\boldsymbol{s}_{*} \neq \boldsymbol{s}$, such that, writing $\boldsymbol{s}$ as a convex combination $\boldsymbol{s}=$ $\mu \boldsymbol{s}_{*}+(1-\mu) \boldsymbol{s}_{* *}(0 \leqslant \mu \leqslant 1)$ of $\boldsymbol{s}_{*}$ and some other point $\boldsymbol{s}_{* *}$, implies that $\mu=0$ (i.e., the combination cannot be proper $)$. Intuitively, this means that straight lines cannot be drawn from the point $s$ in just any direction, if they are to remain inside $S$.

Now, consider a point $s$ given by a convex combination of $\boldsymbol{s}_{j_{1}}$ and $\boldsymbol{s}_{j_{2}}$ :

$$
\boldsymbol{s} \equiv \lambda \boldsymbol{s}_{j_{1}}+(1-\lambda) \boldsymbol{s}_{j_{2}}, \quad 0 \leqslant \lambda \leqslant 1 .
$$

since $\boldsymbol{s}_{j_{1}}, \boldsymbol{s}_{j_{2}}$, and $\boldsymbol{s}_{j_{3}}$ are one-shot distinguishable, there must exist a measurement outcome $\boldsymbol{r}$ such that

$$
\begin{aligned}
& \boldsymbol{r} \cdot \boldsymbol{s}_{j_{1}}=\boldsymbol{r} \cdot \boldsymbol{s}_{j_{2}}=0 \\
& \boldsymbol{r} \cdot \boldsymbol{s}_{j_{3}}=1
\end{aligned}
$$

and Eqs. (A1), (A2) yield

$$
\boldsymbol{r} \cdot \boldsymbol{s}=\lambda \boldsymbol{r} \cdot \boldsymbol{s}_{j_{1}}+(1-\lambda) \boldsymbol{r} \cdot \boldsymbol{s}_{j_{2}}=0 .
$$

Now write $s$ as a convex combination of the vector $\boldsymbol{s}_{j_{3}}$ with some other vector $\boldsymbol{s}_{* *}$ :

$$
\boldsymbol{s}=\mu \boldsymbol{s}_{j_{3}}+(1-\mu) \boldsymbol{s}_{* *}, \quad 0 \leqslant \mu \leqslant 1
$$

From Eqs. (A2), (A4), and (A3), one obtains

$$
0=\boldsymbol{r} \cdot \boldsymbol{s}=\mu \boldsymbol{r} \cdot \boldsymbol{s}_{j_{3}}+(1-\mu) \boldsymbol{r} \cdot \boldsymbol{s}_{* *}=\mu+(1-\mu) \boldsymbol{r} \cdot \boldsymbol{s}_{* *}
$$

which can be satisfied only if $\mu=0$ (which implies $\left.\boldsymbol{s}_{* *}=\boldsymbol{s}\right)$. Thus, the vector $\boldsymbol{s}_{j_{3}}$ plays the role of $\boldsymbol{s}_{*}$ in the condition given above, and so any $\boldsymbol{s}=\lambda \boldsymbol{s}_{j_{1}}+(1-\lambda) \boldsymbol{s}_{j_{2}}$ lies in the boundary of $S$. An analogous proof holds for any convex combination of any two of the vectors $\left\{\boldsymbol{s}_{j_{1}}, \boldsymbol{s}_{j_{2}}, \boldsymbol{s}_{j_{3}}\right\}$, and this implies that the latter are vertices of a triangle which is part of the boundary of $S$.

The generalisation to more than three vectors is straightforward.

\section{Appendix B: THE TRACE RULE}

It is shown that the 'scalar product formula', Eq. (4), includes also the 'trace rule' of quantum mechanics ( $c f$. Hardy [6, Sec. 5]; also Weigert [5]).

A state is usually represented in quantum mechanics by a density matrix $\hat{\boldsymbol{\rho}}_{j}$, and a measurement outcome by a positive-operator-valued measure element $\hat{\boldsymbol{A}}_{i}$; both are Hermitian operators in a Hilbert space of dimension $N$. The probability of obtaining the outcome $\hat{\boldsymbol{A}}_{i}$ for a given measurement on state $\hat{\boldsymbol{\rho}}_{j}$ is given by the trace formula

$$
p_{i j}=\operatorname{tr} \hat{\boldsymbol{A}}_{i} \hat{\boldsymbol{\rho}}_{j}
$$

The Hermitian operators form a linear space of real dimension $K=N^{2}$; one can choose $N^{2}$ linearly independent Hermitian operators $\left\{\hat{\boldsymbol{B}}_{k}\right\}$ as a basis for this linear space. These can also be chosen (basically by GramSchmidt orthonormalisation) to satisfy

$$
\operatorname{tr} \hat{\boldsymbol{B}}_{k} \hat{\boldsymbol{B}}_{l}=\delta_{k l} \text {. }
$$

Both $\hat{\boldsymbol{\rho}}_{j}$ and $\hat{\boldsymbol{A}}_{i}$ can be written as a linear combination of the basis states

$$
\hat{\boldsymbol{\rho}}_{j}=\sum_{l=1}^{K} s_{j}{ }^{l} \hat{\boldsymbol{B}}_{l}, \quad \hat{\boldsymbol{A}}_{i}=\sum_{k=1}^{K} r_{i}{ }^{k} \hat{\boldsymbol{B}}_{k}
$$

where the coefficients $s_{j}{ }^{l}$ and $r_{i}{ }^{k}$ are real. Using Eqs. (B3) and (B2) the trace formula becomes

$p_{i j}=\operatorname{tr} \hat{\boldsymbol{A}}_{i} \hat{\boldsymbol{\rho}}_{j}=\sum_{k, l=1}^{K} r_{i}{ }^{k} s_{j}{ }^{l} \operatorname{tr} \hat{\boldsymbol{B}}_{k} \hat{\boldsymbol{B}}_{l}=\sum_{l=1}^{K} r_{i}{ }^{l} s_{j}{ }^{l}=\boldsymbol{r}_{i} \cdot \boldsymbol{s}_{j}$,

where $\boldsymbol{r}_{i} \stackrel{\text { def }}{=}\left(r_{i}{ }^{1} \ldots r_{i}{ }^{K}\right)$ and $\boldsymbol{s}_{j} \stackrel{\text { def }}{=}\left(s_{j}{ }^{1} \ldots s_{j}{ }^{K}\right)$ are vectors in $\mathbb{R}^{K}$.
[1] E. Wigner, Phys. Rev. 40, 749 (1932)

[2] H. P. Stapp, Phys. Rev. D 3, 1303 (1971).

[3] W. K. Wootters, Found. Phys. 16, 391 (1986).

[4] W. K. Wootters, Annals of Physics 176, 1 (1987).

[5] S. Weigert, Phys. Rev. Lett. 84, 802 (2000), quant$\mathrm{ph} / 9903103$.

[6] L. Hardy, Quantum theory from five reasonable axioms (2001), quant-ph/0101012.

[7] T. F. Havel, The real density matrix (2003), quant$\mathrm{ph} / 0302176$.

[8] A. Peres, Quantum Theory: Concepts and Methods, vol. 72 of Fundamental Theories of Physics (Kluwer Academic Publishers, Dordrecht, 1995).

[9] A. Peres and D. R. Terno, J. Phys. A: Math. Gen. 31, L671 (1998).

[10] P. Busch and P. J. Lahti, Phys. Rev. D 29, 1634 (1984).

[11] P. Busch, Phys. Rev. D 33, 2253 (1986).

[12] H. Barnum, Tech. Rep. LA-UR 03-1199, Los Alamos Na- tional Laboratory (2003), quant-ph/0304159.

[13] H. S. M. Coxeter, Regular Polytopes (Dover, New York, 1948), 3rd ed.

[14] G. Kimura, The Bloch vector for N-level systems (2003), quant-ph/0301152.

[15] L. Jakóbczyk and M. Siennicki, Phys. Lett. A 286, 383 (2001).

[16] P. Pechukas, Phys. Rev. Lett. 73, 1060 (1994).

[17] P. Štelmachovič and V. Bužek, Phys. Rev. A 64, 062106 (2001), quant-ph/0108136.

[18] A. Peres and D. R. Terno, Quantum information and relativity theory (2002), quant-ph/0212023.

[19] A. Peres and D. R. Terno, Quantum information and special relativity (2002), quant-ph/0301065.

[20] J. Tissot, Discours de la vie et mort du géant Teutobochus, Lyon? (1613?); seemingly cited in É. Fournier, L'Esprit des autres (Dentu, Paris, 1857), 3rd ed., and in W. F. H. King, Classical and foreign quotations: A 
polyglot dictionary of historical and literary quotations, proverbs, and popular sayings (J. Whitacker and Sons, London, 1904).

[21] C. von Linné (Carolus Linnaeus), Philosophia botanica in qva explicantur fundamenta botanica cum definitionibus partium, exemplis terminorum, observationibus rariorum, adjectis figuris aeneis. (Grefing, Stockholm, 1751).

[22] H. Jeffreys, Scientific Inference (Cambridge University Press, London, 1931), 2nd ed.

[23] K. A. Kirkpatrick, "Quantal" behavior in classical prob- ability (2001), quant-ph/0106072.

[24] K. A. Kirkpatrick, Classical three-box "paradox" (2002), quant-ph/0207124.

[25] K. A. Kirkpatrick, Hardy's second axiom is insufficiently general (2003), quant-ph/0302158.

[26] P. G. L. Mana, The properties of the Shannon entropy are not violated in quantum measurements (2003), quantph/0302049. 